\title{
COMPATIBLE TIGHT RIESZ ORDERS ON $C(X)$
}

\author{
ELIZABETH LOCI
}

(Received 10 March 1975; revised 16 September 1975)

\begin{abstract}
The pointwise order makes the group $C(X)$ of continuous real-valued functions on a topological space $X$ a lattice-ordered group. We give a characterization of the compatible tight Riesz orders on $C(X)$, and also of their maximal tangents, in terms of the zero-sets of $X$. The space of maximal tangents of a given compatible tight Riesz order $T$ is studied, and consequently the concept of the $T$-radical of $C(X)$ is introduced, the $T$-radical being the intersection of all the maximal tangents of $T$.
\end{abstract}

\section{Introduction}

Given a topological space $X$ we denote by $C(X)$ the set of continuous real-valued functions on $X$. If $C(X)$ is equipped with the following order

$$
f \geqslant 0 \text { if } f(x) \geqq 0 \text { for all } x \in X
$$

then it becomes an abelian lattice-ordered group with

$$
\begin{aligned}
& f \wedge g(x)=\min \{f(x), g(x)\} \\
& f \vee g(x)=\max \{f(x), g(x)\} .
\end{aligned}
$$

In order to obtain the compatible tight Riesz orders on $C(X)$, we make use of the following result due essentially to Wirth (1973). A compatible tight Riesz order on a lattice-ordered group $(G, \leqslant)$ is determined by (and determines) a subset $T$ of the positive set $G^{+}=\{x \in G: 0 \leqslant x\}$ of $G$ satisfying the following conditions:

(1) $T$ is a proper dual-ideal of $G^{+}$

(2) $T$ is normal in $G$

(3) $T=T+T$

(4) $0 \leqslant n x \leqslant y$ for all positive integers $n$, for all $y \in T$, implies $x=0$.

In the case of $C(X)$ we can modify the above result, since, $C(X)$ abelian implies (2) holds for all subsets $T$ of $C^{+}(X), \mathbb{R}$ archimedean implies (4) holds for all 
subsets $T$ of $C^{+}(X)$ and $C(X)$ divisible makes (3) easier to check. We have then that the compatible tight Riesz orders on $C(X)$ are determined by (and determine) proper dual-ideals $T$ of $C^{+}(X)$ satisfying $T=T+T$. By an abuse of language we shall call each such dual-ideal $T$ a compatible tight Riesz order on $C(X)$.

With each compatible tight Riesz order $T$ on $C(X)$ we associate tangents [cf. Miller (1973)] i.e. convex sublattice subgroups of $C(X)$ not meeting $T$ and maximal tangents i.e. convex sublattice subgroups of $C(X)$ that are maximal with respect to not meeting $T$. Each maximal tangent $M$ of $T$ satisfies the further condition $-f \wedge g \in M$ implies $f \in M$ or $g \in M$ - i.e. each maximal tangent is a prime subgroup of $C(X)$. Finally, if $C(X)$ is given the open-interval topology generated by the compatible tight Riesz order $T$, then every tangent of $T$ is closed.

\section{Zero-set characterization of compatible tight Riesz orders}

We proceed in analogy to Gillman and Jerison (1960).

Given $f \in C(X)$, the set $\{x \in X: f(x)=0\}$ is called the zero-set of $f$, and will be denoted by $Z(f)$. Any set that is a zero-set of some function in $C(X)$ is called a zero-set in $X$, and we denote the set of all zero-sets in $X$ by $Z(X)$. Now

$$
Z(f) \cup Z(g)=Z(|f|) \cup Z(|g|)=Z(|f| \wedge|g|)
$$

and

$$
Z(f) \cap Z(g)=Z(|f|) \cap Z(|g|)=Z(|f| \vee|g|)
$$

whence $Z(X)$ is closed under finite unions and intersections. Thus $Z(X)$, ordered by inclusion, is a lattice, and we make the following (usual) definitions -

A non-empty subfamily $\mathscr{F}$ of $Z(X)$ is called a $Z$-ideal provided that:

(1) if $Z_{1}, Z_{2} \in \mathscr{F}$ then $Z_{1} \cup Z_{2} \in \mathscr{F}$

(2) if $Z \in \mathscr{F}, Z^{\prime} \in Z(X)$ and $Z \supset Z^{\prime}$ then $Z^{\prime} \in \mathscr{F}$

If in addition

(3) $X \notin \mathscr{F}$

then $\mathscr{F}$ is a proper $Z$-ideal.

A non-empty subfamily $\mathscr{G}$ of $Z(X)$ is called a $Z$-filter provided that:

(1) if $Z_{1}, Z_{2} \in \mathscr{G}$ then $Z_{1} \cap Z_{2} \in \mathscr{G}$

(2) if $Z \in \mathscr{G}, Z^{\prime} \in Z(X)$ and $Z^{\prime} \supset Z$ then $Z^{\prime} \in \mathscr{G}$.

If in addition

(3) $\square \notin \mathscr{G}$

then $\mathscr{G}$ is a proper $Z$-filter.

Throughout this paper we will assume all $Z$-ideals and $Z$-filters to be proper. 
THEOREM 1. (a) If $T$ is a proper dual-ideal of $C^{+}(X)$, then the family

$$
Z[T]=\{Z(f): f \in T\}
$$

is a Z-ideal.

(b) If $\mathscr{F}$ is a $Z$-ideal then the family

$$
Z \leftarrow[\mathscr{F}]^{*}=\{|f|: Z(f) \in \mathscr{F}\}
$$

is a proper dual-ideal of $C^{+}(X)$.

Proof. (a) 1 . Let $Z_{1}, Z_{2} \in Z[T]$. Choose $f_{1}, f_{2} \in T$ satisfying $Z_{1}=Z\left(f_{1}\right)$, $Z_{2}=Z\left(f_{2}\right)$, then since $f_{1}, f_{2} \in C^{+}(X)$ we have $Z\left(f_{1}\right) \cup Z\left(f_{2}\right)=Z\left(f_{1} \wedge f_{2}\right)$, and since $T$ is a dual-ideal, $f_{1} \wedge f_{2} \in T$. Thus $Z_{1} \cup Z_{2} \in Z[T]$.

2. Let $Z \in Z[T]$ and $Z^{\prime} \in Z(X)$ with $Z \supset Z^{\prime}$. Choose $f \in T$ and $f^{\prime} \in C(X)$ satisfying $\quad Z=Z(f), \quad Z^{\prime}=Z\left(f^{\prime}\right)=Z\left(\left|f^{\prime}\right|\right)$. Then $Z(f) \supset Z\left(\left|f^{\prime}\right|\right)$ whence $Z\left(f \vee\left|f^{\prime}\right|\right)=Z(f) \cap Z\left(\left|f^{\prime}\right|\right)=Z\left(\left|f^{\prime}\right|\right)=Z^{\prime}$. But $f \leqslant f \vee\left|f^{\prime}\right|$ implies $f \vee\left|f^{\prime}\right| \in T$, thus $Z^{\prime} \in Z[T]$.

3. $T$ proper implies $0 \notin T$ implies $X \notin Z[T]$.

(b) 1. Let $f=|f|, \quad g=|g| \in Z \leftarrow[\mathscr{F}]^{*}$. Then $f \wedge g=|f \wedge g|$ and $Z(f \wedge g)=Z(f) \cup Z(g) \in \mathscr{F}$ whence $f \wedge g \in Z \leftarrow[\mathscr{F}]^{*}$.

2. Let $f=|f| \in Z \leftarrow[\mathscr{F}]^{*}$ and let $g \in C(X)$ satisfy $f \leqslant g$, whence $g \in C^{+}(X)^{+}(X)$. Now $f, g \in C^{+}(X), f \leqslant g$ imply $Z(f) \supset Z(g)$ whence $Z(g) \in \mathscr{F}$. Thus $g=|g| \in Z \leftarrow[\mathscr{F}]^{*}$.

3. $X \notin \mathscr{F}$ implies $0 \notin Z \leftarrow[\mathscr{F}]^{*}$.

It is worth noting that since $C(X)$ is divisible we have that $Z(f)=Z(f / 2)$ for all $f \in C(X)$. In particular then, given $\mathscr{F}$ a $Z$-ideal and $f=|f| \in Z \leftarrow[\mathscr{F}]^{*}$ we have $f / 2 \in Z \leftarrow[\mathscr{F}]^{*}$. Thus, Theorem 1 may be read with 'compatible tight Riesz order on $C(X)$ ' for 'proper dual-ideal of $C^{+}(X)$ '.

Clearly we have the following relationships

$$
Z\left[Z \leftarrow[\mathscr{F}]^{*}\right]=\mathscr{F}
$$

and

$$
Z \leftarrow[Z[T]]^{*} \supset T
$$

for all $Z$-ideals $\mathscr{F}$ and compatible tight Riesz orders $T$. A compatible tight Riesz order $T$ satisfying $Z \leftarrow[Z[T]]^{*}=T$ will be called an algebraic tight Riesz order.

If $\mathscr{F}$ is a $Z$-ideal then $Z \leftarrow[\mathscr{F}]^{*}$ is an algebraic tight Riesz order. Every maximal compatible tight Riesz order is algebraic.

Using the term adjunction in the sense of MacLane (1971), we may restate our previous results as follows: 
THEOREM 2. There is an adjunction $Z \vdash Z \leftarrow{ }^{*}$ from the set of compatible tight Riesz orders on $C(X)$, ordered by inclusion, to the set of $Z$-ideals of $Z(X)$, ordered by inclusion, such that the algebras for this adjunction are just the algebraic tight Riesz orders.

COROllary 3. The unique minimal algebraic tight Riesz order is $T_{0}=$ $\{f \in C(X): f(x)>0$ for all $x \in X\}$.

Proof. The unique minimal $Z$-ideal is $\{\square\}$ and $Z \leftarrow[\{\square\}]^{*}=T_{0}$.

COROLlaRy 4. $T_{0}$ is contained in every algebraic tight Riesz order. Corollaries 3 and 4 are in fact special cases of a result of Wirth (1973, Lemma 4).

THEOREM 5. If $T$ is an algebraic tight Riesz order on $C(X)$ there is an adjunction $Z^{T}+Z \leftarrow^{* T}$ from the set of convex sublattice subgroups of $C(X)$ not meeting $T$, ordered by inclusion, to the set of $Z$-filters of $Z(X)$ not meeting $Z[T]$, ordered by inclusion, such that the algebras for this adjunction include the maximal tangents of $T$.

Proof. Let $T$ be an algebraic tight Riesz order and $G$ a convex sublattice subgroup (vector lattice ideal) of $C(X)$ not meeting $T$. Consider $Z[G]$.

(1) $\square \notin Z[G]$, for suppose otherwise, then there exists $f \in G$ such that $f(x) \neq 0$ for all $x \in X$ i.e. $|f| \in G \cap T_{0}$, a contradiction, since by Corollary $4, T_{0} \subset T$. (2) Let $Z_{1}, Z_{2} \in Z[G]$. Choose $f, g \in G \cap C^{+}(X)$ such that $Z_{1}=Z(f)$ and $Z_{2}=Z(g)$. Then $Z_{1} \cap Z_{2}=Z(f \vee g) \in Z[G]$ since $G$ is a sublattice. Thus $Z[G]$ is a filterbase and we denote the $Z$-filter generated by $Z[G]$ by $Z[G]^{T}$, i.e. $Z[G]^{T}=\{Z \in Z(X): Z \supset Z(f)$ for some $f \in G\}$. Suppose $Z[G]^{T}$ meets $Z[T]$. Then there exists $f \in T, g \in G$ such that $Z(f) \supset Z(g)$. But $T$ an algebraic tight Riesz order and $Z(f) \supset Z(g)$ imply $|g| \in T$, i.e. $|g| \in G \cap T$, a contradiction. Thus $Z[G]^{T}$ is a $Z$-filter not meeting $Z[T]$.

Conversely, let $\mathscr{F}$ be a $Z$-filter not meeting $Z[T]$. Consider $N=Z \leftarrow[\mathscr{F}]^{*}$.

(1) Given $g \in N$ and $0 \leqslant f \leqslant g$ we have $Z(f) \supset Z(g)$ whence $Z(f) \in \mathscr{F}$, i.e. $N$ is convex.

(2) Given $f, g \in N$ we have that $f \wedge g \in N$ since $Z(f \wedge g)=Z(f) \cup Z(g)$ $\supset Z(g) \in \mathscr{F}$ and that $f \vee g \in N$ since $Z(f \vee g)=Z(f) \cap Z(g) \in \mathscr{F}$. Thus $N$ is a sublattice.

(3) Moreover $(N,+)$ is a subsemigroup of $C(X)$, since given $f, g \in N$ we have $Z(f+g)=Z(f) \cap Z(g) \in \mathscr{F}$.

(4) $N \cap T=\square$ for if $f \dot{E} N \cap T$ then $Z(f) \in \mathscr{F} \cap Z[T]$, a contradiction. Thus $N$ is a convex sublattice subsemigroup not meeting $T$. Remembering that every directed subgroup is generated by its positive elements we have that $Z \leftarrow[\mathscr{F}]^{* T}=\{f-g: f, g \in N\}$ is a convex sublattice subgroup not meeting $T$. 
Recall that $Z \leftarrow\left[Z\left[T^{\prime}\right]\right]^{*} \supset T^{\prime}$ for all dual-ideals $T^{\prime}$ of $C^{+}(X)$. Similarly $Z \leftarrow[Z[G]]^{* T} \supset G$ for all convex sublattice subgroups of $C(X)$ not meeting $T$. Thus $Z \leftarrow[Z[M]]^{* T}=M$ for all maximal tangents of $T$.

COROllary 6. If $T$ is an algebraic tight Riesz order on $C(X)$ there is a one-one correspondence between maximal tangents of $T$ and $Z$-filters maximal with respect to not meeting $Z[T]$.

The following result is due to Gillman and Jerison (1960),

THEOREM 7. If $\mathscr{F}$ is a $Z$-filter, then the family $Z \leftarrow[\mathscr{F}]=\{f: Z(f) \in \mathscr{F}\}$ is a ring-ideal of $C(X)$.

Using this criterion for obtaining ring-ideals of $C(X)$ we prove the following:

THEOREM 8. If $\boldsymbol{F}$ is an algebraic tight Riesz order then each maximal tangent of $T$ is a ring-ideal of $C(X)$.

Proof. Let $T$ be an algebraic tight Riesz order and let $M$ be a maximal tangent of $T$. Corollary 6 tells us that $Z[M]^{T}=\{Z \in Z(X): Z \supset Z(f)$ for some $f \in M\}$ is a $Z$-filter and moreover that $Z(g)=Z(f)$ for some $f \in M$ implies $|g| \in M$.

Consider $Z \leftarrow\left[Z[M]^{T}\right]=\{g: Z(g) \supset Z(f)$ for some $f \in M\}$. Now

$$
\begin{aligned}
g \in Z \leftarrow\left[Z[M]^{T}\right] & \Rightarrow Z(|g|) \cap Z(|f|)=Z(f) \text { for some } f \in M \\
& \Rightarrow|g| \vee|f| \in M \\
& \Rightarrow|g| \text { whence } g \in M, M \text { a convex subgroup. }
\end{aligned}
$$

Thus $Z \leftarrow\left[Z[M]^{T}\right] \subset M$. The converse is trivially true, so by Theorem $7, M$ is a ring-ideal of $C(X)$.

Given $T$ an algebraic tight Riesz order on $C(X)$ we define the $T$-radical of $C(X)$ to be the intersection of all the maximal tangents of $T$. Clearly the $T$-radical of $C(X)$ is a ring-ideal of $C(X)$.

\section{The space of maximal tangents of an algebraic tight Riesz order}

Let $T$ be an algebraic tight Riesz order. The set of all maximal tangents of $T$ is denoted by $\operatorname{Max}(T)$. Given $f \in C^{+}(X)$ define

$$
U(f)=\{M \in \operatorname{Max}(T): f \notin M\} .
$$

Then we have the following:

Lemma 9. $\left\{U(f): f \in C^{+}(X)\right\}$ is a base topology, say $U$, on $\operatorname{Max}(T)$. 
Proof. (1) $f \in T$ implies $U(f)=\operatorname{Max}(T)$, so that $\operatorname{Max}(T)=$ $\cup\left\{U(f): f \in C^{+}(X)\right\}$.

(2) Let $M \in \operatorname{Max}(T)$, and $f_{1}, f_{2} \in C^{+}(X)$. Now $f_{1} \in M$ or $f_{2} \in M$ implies $f_{1} \wedge f_{2} \in M(M$ convex $)$ whence $f_{1} \wedge f_{2} \notin M$ implies $f_{1} \notin M$ and $f_{2} \notin M$, i.e. $U\left(f_{1} \wedge f_{2}\right) \subseteq U\left(f_{1}\right) \cap U\left(f_{2}\right)$. Conversely, $f_{1} \wedge f_{2} \in M$ implies $f_{1} \in M$ or $f_{2} \in M$ ( $M$ prime) whence $f_{1} \notin M$ and $f_{2} \notin M$ imply $f_{1} \wedge f_{2} \notin M$, i.e. $U\left(f_{1}\right) \cap U\left(f_{2}\right) \subset$ $U\left(f_{1} \wedge f_{2}\right)$.

Thus $U\left(f_{1}\right) \cap U\left(f_{2}\right)=U\left(f_{1} \wedge f_{2}\right)$.

Similarly to (2) in the above proof we can show $U\left(f_{1}\right) \cup U\left(f_{2}\right)=U\left(f_{1} \vee f_{2}\right)$.

Proposition 10. $(\operatorname{Max}(T), U)$ is a $T_{1}$-space.

Proof. Let $M_{1}$ and $M_{2}$ be distinct members of $\operatorname{Max}(T)$. Then there exist $f_{1} \in\left(M_{2} \cap C^{+}(X)\right) \backslash M_{1}$ and $f_{2} \in\left(M_{1} \cap C^{+}(X)\right) \backslash M_{2}$, i.e. $M_{1} \in U\left(f_{1}\right), M_{2} \notin U\left(f_{1}\right)$ and $M_{2} \in U\left(f_{2}\right), M_{1} \notin U\left(f_{2}\right)$.

Proposition 11. (Max $(T), U)$ is compact.

Proof. Basic closed sets being complements of basic open sets are of the form $V(f)=\{M \in \operatorname{Max}(T): f \in M\}, f \in C^{+}(X)$. Let $\left\{V\left(f_{\lambda}\right): \lambda \in \Lambda\right\}$ be a collection of basic closed sets with the finite intersection property, i.e. $V\left(f_{\lambda_{2}}\right) \cap \cdots \cap$ $V\left(f_{\lambda_{n}}\right) \neq \square$ for all finite subsets $\left\{\lambda_{1}, \cdots, \lambda_{n}\right\}$ of $\Lambda$.

Consider $I \cdots$ the ideal generated by $\left\{f_{\lambda}\right\}_{\lambda \in \Lambda}$. $I$ does not meet $T$, for if so there exists $g \in T$ such that $g \leqslant f_{\lambda_{1}} \vee \cdots, \vee f_{\lambda_{n}}$ for some $\lambda_{1}, \cdots, \lambda_{n} \in \Lambda$. But then $f_{\lambda_{1}} \vee \cdots \vee f_{\lambda_{n}} \in T$ so that $V\left(f_{\lambda_{1}} \vee \cdots \vee f_{\lambda_{n}}\right)=\square$. However $V\left(f_{\lambda_{1}} \vee \cdots \vee f_{\lambda_{n}}\right)=$ $V\left(f_{\lambda_{1}}\right) \cap \cdots \cap V\left(f_{\lambda_{n}}\right) \neq \square$ so that $I$ is a proper $l$-ideal containing $\left\{f_{\lambda}\right\}_{\Lambda \in \Lambda}$ and not meeting $T$.

Suppose $\bigcap_{\lambda \in A} V\left(f_{A}\right)=\square$. This says that there exists no $l$-ideal containing all the $f_{\lambda}$ 's and not meeting $T$. This however is clearly false, since $I$ meets all these requirements. Thus $\bigcap_{\lambda \in \Lambda} V\left(f_{\wedge}\right) \neq \square$, whence $(\operatorname{Max}(T), U)$ is compact.

Proposition 12. Let $T$ and $T^{\prime}$ be algebraic tight Riesz orders such that $T \subseteq T^{\prime}$. Then each maximal tangent of $T^{\prime}$ is contained in a unique maximal tangent of $T$.

Proof. Let $M^{\prime} \in \operatorname{Max}\left(T^{\prime}\right)$. Then $M^{\prime}$ is a prime subgroup not meeting $T$. Since the class of convex sublattice subgroups lying above a prime subgroup is totally-ordered by inclusion [Holland (1963)] we have that $\boldsymbol{M}^{\prime}$ is contained in a unique maximal tangent $M$ of $T$.

Theorem 13. Let $T$ and $T^{\prime}$ be algebraic tight Riesz orders such that $T \subseteq T^{\prime}$. If $\left(\operatorname{Max}\left(T^{\prime}\right), U^{\prime}\right)$ is Hausdorf then the map $m:\left(\operatorname{Max}\left(T^{\prime}\right), U^{\prime}\right) \rightarrow(\operatorname{Max}(T), U)$ 
given by $m\left(M^{\prime}\right)=M-$ the unique maximal tangent of $T$ containing $M^{\prime}-$ is continuous.

Proof. Given a basic open set $U(f)=\{M \in \operatorname{Max}(T): f \notin M\}$ we want to see that $S=\left\{M^{\prime} \in \operatorname{Max}\left(T^{\prime}\right): m\left(M^{\prime}\right) \in U(f)\right\}$ is open in $\left(\operatorname{Max}\left(T^{\prime}\right), U^{\prime}\right)$, and we do so by seeing that $\operatorname{Max}\left(T^{\prime}\right) \backslash S$ is compact.

Let $\left\{\operatorname{Max}\left(T^{\prime}\right) \backslash U^{\prime}\left(f_{\lambda}\right) \cap \operatorname{Max}\left(T^{\prime}\right) \backslash S: \lambda \in \Lambda\right\}$ be a collection of basic closed subsets of $\operatorname{Max}\left(T^{\prime}\right) \backslash S$ with the finite intersection property, where $U^{\prime}\left(f_{\lambda}\right)=$ $\left\{M^{\prime} \in \operatorname{Max}\left(T^{\prime}\right): f_{\lambda} \notin M^{\prime}\right\}$ for $f_{\lambda} \in C^{+}(X)$. Then for each finite subset $\left\{\lambda_{1}, \cdots, \lambda_{n}\right\}$ of $\Lambda$ we have

$$
S \cup U^{\prime}\left(f_{\lambda_{1}}\right) \cup \cdots \cup U^{\prime}\left(f_{\lambda_{n}}\right) \neq \operatorname{Max}\left(T^{\prime}\right)
$$

If $f \vee f_{\lambda_{1}} \vee \cdots \vee f_{\lambda_{n}} \in T$ for some finite subset $\left\{\lambda_{1}, \cdots, \lambda_{n}\right\}$ of $\Lambda$ then $U\left(f \vee f_{\lambda_{1}} \vee \cdots \vee f_{\lambda_{n}}\right)=U(f) \cup U\left(f_{\lambda_{1}}\right) \cup \cdots \cup U\left(f_{\lambda_{n}}\right)=\operatorname{Max}(T)$. By assumption (equation (1)) there exists $M^{\prime} \in \operatorname{Max}\left(T^{\prime}\right)$ such that $M^{\prime} \notin S$ and $M^{\prime} \notin U^{\prime}\left(f_{\lambda_{i}}\right)$, $i=1,2, \cdots, n$. Then $m\left(M^{\prime}\right) \notin U(f)$ so that $m\left(M^{\prime}\right) \in U\left(f_{\lambda_{i}}\right)$ for some $i$, which implies $M^{\prime} \in U^{\prime}\left(f_{\lambda_{i}}\right)$ - a contradiction. Thus, for every finite subset $\left\{\lambda_{1}, \cdots, \lambda_{n}\right\}$ of $\Lambda, f \vee f_{\lambda_{1}} \vee \cdots \vee f_{\lambda_{n}} \notin T$. Similarly, for every finite subset $\left\{\lambda_{1}, \cdots, \lambda_{n}\right\}$ of $\Lambda$, $f_{\lambda_{1}} \vee \cdots \vee f_{\lambda_{n}} \notin T^{\prime}$. Hence, since by both (Max $\left.(T), U\right)$ and $\left(\operatorname{Max}\left(T^{\prime}\right), U^{\prime}\right)$ are compact (Proposition 11), there is an $M^{\prime} \in \operatorname{Max}\left(T^{\prime}\right)$ containing all $f_{\lambda}: \lambda \in \Lambda$, and an $M \in \operatorname{Max}(T)$ containing $M^{\prime}$ and $f$. Then $m\left(M^{\prime}\right)=M$, so we have $M^{\prime} \notin S$ and $M^{\prime} \notin U^{\prime}\left(f_{\lambda}\right)$, for all $\lambda \in \Lambda$, i.e. $\operatorname{Max}\left(T^{\prime}\right) \backslash S$ is compact.

In proving the above theorem we made use of the fact that ( $\left.\operatorname{Max}\left(T^{\prime}\right), U^{\prime}\right)$ was Hausdorff. We now consider necessary and sufficient conditions for such a space to be Hausdorff.

Proposition 14. $(\operatorname{Max}(T), U)$ is Hausdorff if and only if given $M_{1}$ and $M_{2}$ distinct members of $\operatorname{Max}(T)$ there exist $f_{1} \in C^{+}(X) \backslash M_{1}$ and $f_{2} \in C^{+}(X) \backslash M_{2}$ such that $f_{1} \wedge f_{2} \in T$-radical of $C(X)=\cap\{M: M \in \operatorname{Max}(T)\}$.

The proof is obvious.

THEOREM 15. $M_{1}$ and $M_{2}$ distinct members of $\operatorname{Max}(T)$ can be Hausdorff separated for $U$ if either $M_{1}$ or $M_{2}$ is minimal prime.

Proof. $M_{1}$ and $M_{2}$ distinct implies that there exist $f_{1} \in\left(M_{2} \cap C^{+}(X)\right) \backslash M_{1}$ and $f_{2} \in\left(M_{1} \cap C^{+}(X)\right) \backslash M_{2}$. Suppöse $M_{2}$ is minimal prime. Then there exists $f \in C^{+}(X) \backslash M_{2}$ such that $f_{1} \wedge f=0$. Moreover $f \notin M_{2}$ implies $f \wedge f_{2} \in M_{1} \backslash M_{2} \cap$ $C^{+}(X)$ (primality). Thus $U\left(f_{1}\right) \cap U\left(f \wedge f_{2}\right)=U\left(f_{1} \wedge f \wedge f_{2}\right)=U(0)=\square . \quad$ A similar argument holds if $M_{1}$ is minimal prime.

THEOREM 16. If $T$ is dual-prime then $(\operatorname{Max}(T), U)$ is a singleton.

Proof. Let $M \in \operatorname{Max}(T)$. Then 


$$
\begin{aligned}
f \notin M & \Leftrightarrow|f| \vee|g| \in T \text { for some } g \in M \\
& \Leftrightarrow|f| \in T \quad(T \text { dual-prime })
\end{aligned}
$$

i.e. there is but one maximal tangent of $T$.

\section{The quotient space $C(X) / A$}

Thoughout this section we assume $X$ to be a compact Hausdorff space and $T$ to be an algebraic tight Riesz order on $C(X)$. We denote the $T$-radical of $C(X)$ by $A$ i.e. $A=\cap\{M: M \in \operatorname{Max}(T)\}$.

As a result of Proposition 14, we see that $A$ plays an important role in determining whether or not $(\operatorname{Max}(T), U)$ is Hausdorff. For this reason we make a brief study of $A$ and consequently of the quotient space $C(X) / A$.

Being an intersection of maximal tangents of $T, A$ is a tangent - hence an $l$-ideal (not necessarily prime), and so we may consider $C(X) / A$ as the factor group of $C(X)$ with respect to the $l$-ideal $A$. Then $C(X) / A$ is a lattice-ordered group and the canonical mapping $\rho: C(X) \rightarrow C(X) / A$ preserves the order relation and lattice operations, (Fuchs (1963)). We use the same symbol $\leqslant$ to denote the lattice-order in both $C(X)$ and $C(X) / A$, and we denote $\{f+A: 0+$ $A \leqslant f+A\}$ by $C(X) / A^{+}$, where we have $0+A \leqslant f+A$ if and only if $0 \leqslant f+a$ for some $a \in A$.

We consider the action of the canonical mapping $\rho: C(X) \rightarrow C(X) / A$.

TheOREM 17. $\rho T$ is a compatible tight Riesz order on $C(X) / A$.

Proof. This follows immediately from Theorem $8^{\circ}$ of Miller (1973).

THEOREM 18. Let $M$ be a maximal tangent of $T$ then $\rho M$ is a maximal tangent of $\rho T$.

Proof. Put $M^{\prime}=\rho M=M+A, T^{\prime}=\rho T=T+A$. Then

(1) Since $\rho$ preserves the order relation and lattice operations we have immediately that $M^{\prime}$ is a convex sublattice of $C(X) / A$.

(2) $M^{\prime}$ is non-empty since $M$ is non-empty. Moreover, it is straightforward to show that $M^{\prime}$ is closed under addition and that each element in $M^{\prime}$ has an additive inverse in $M^{\prime}$. Thus $M^{\prime}$ is a subgroup of $C(X) / A$.

(3) Suppose $f+A \in M^{\prime} \cap T^{\prime}$. Now $f+A \in M+A$ implies $f+A=m+A$ for some $m \in M$ i.e. $f-m \in A \subset M$ for some $m \in M$. In other words $f \in M$. Similarly $f+A \in T+A$ implies $f-t \in M$ for some $t \in T$. Then $f^{*}(f-t)=$ $t \in M$ for some $t \in T$, a contradiction. Thus $M^{\prime} \cap T^{\prime}=\square$.

(4) Suppose $M^{\prime} \subset N^{\prime}$ where $N^{\prime}$ is a convex sublattice subgroup of $C(X) / A$ not meeting $T^{\prime}$. Put $N=\left\{f: f+A \in N^{\prime}\right\}$. Then $N$ is a convex sublattice subgroup of 
$C(X)$ not meeting $T$. Moreover $M \subset N$. Thus $M=N$ and $M^{\prime}=N^{\prime}$ since $M$ is a maximal tangent of $T$.

In other words, $M^{\prime}$ is a maximal tangent of the compatible tight Riesz order $T^{\prime}$.

Corollary 19. Let $\mathcal{M}$ be the set of maximal tangents of $\rho T$, then $\cap$ $\{M ; M \in \mathcal{M}\}=0+A$.

Proof. This follows since $\rho$ preserves intersections.

\section{References}

L. Fuchs (1963), Partially Ordered Algebraic Systems (Pergamon Press, Oxford, 1963).

L. Gillman and M. Jerison (1960), Rings of Continuous Functions (Van Nostrand, Princeton, 1960).

C. Holland (1963), 'The lattice-ordered group of automorphisms of an ordered set', Mich. Math J. 10, $399-408$.

Saunders Maclane (1971), Categories for the Working Mathematician (Springer-Verlag, New York, 1971).

J. B. Miller (1973), 'Quotient groups and realization of tight Riesz groups', J, Austral. Math. Soc. 16, $416-430$.

A. Wirth (1973), 'Compatible tight Riesz orders', J. Austral. Math Soc. 15, 105-111.

Department of Mathematics,

La Trobe University,

Victoria 3083,

Australia. 